Esta revista forma parte del acervo de la Biblioteca Jurídica Virtual del Instituto de Investigaciones Jurídicas de la UNAM

\title{
TRANSPARENCIA EN LOS AYUNTAMIENTOS: DISCRECIONALIDAD EN SU INTEGRACIÓN Y REMUNERACIONES
}

TRANSPARENCY IN TOWN HALLS: DISCRETION IN ITS INTEGRATION AND REMUNERATIONS

\author{
Jacqueline PESCHARD MARISCAL* \\ Grisel SALAZAR REBOLLEDO** \\ Octavio OLEA GÓMEZ***
}

\begin{abstract}
RESUMEN. ¿Se ha arraigado la cultura de la transparencia en el gobierno municipal? ¿Es la opacidad un recurso para manejar de manera discrecional cargos y recursos en ese nivel de gobierno? El objetivo de este artículo es dar cuenta del estado de la transparencia en dos aspectos básicos del gobierno municipal en México: la integración del cabildo y la remuneración de sus miembros. Desde la teoría, el municipio ha sido concebido como el espacio más cercano a las demandas de la ciudadanía, donde surgen y se ejercen las virtudes democráticas. Sin embargo, lo que muestran los datos es que en algunos municipios persisten claras características de arbitrariedad y opacidad. En este artículo se estudian los municipios de los estados que celebraron elecciones en 2017
\end{abstract}

* Doctora en ciencias sociales por El Colegio de Michoacán, maestra en ciencias políticas y licenciada en sociología por la Facultad de Ciencias Políticas y Sociales (FCPyS) de la UNAM. Es profesora investigadora de la FCPyS y coordinadora del Seminario Universitario de Transparencia de la UNAM, jacpeschard@yahoo.com.mx.

** Doctora en políticas públicas por el Centro de Investigación y Docencia Económicas (CIDE), maestra en ciencia política por la Universidad de Salamanca, España, y licenciada en política y administración pública por El Colegio de México. Es profesora asociada del CIDE, y profesora de asignatura en la FCPyS, e investigadora del Seminario Universitario de Transparencia de la UNAM, maria.salazar@cide.edu.

*** Maestro en estudios políticos y sociales por la FCPyS de la UNAM, y licenciado en ciencias políticas y administración pública por la misma institución. Es secretario técnico del Seminario Universitario de Transparencia de la UNAM, augustoolea@gmail.com.

Fecha de recepción: 8 de enero de 2018.

Fecha de dictamen: 8 de febrero de 2018 . 
Esta revista forma parte del acervo de la Biblioteca Jurídica Virtual del Instituto de Investigaciones Jurídicas de la UNAM

—Coahuila, Estado de México, Nayarit y Veracruz-, específicamente sobre la integración y las remuneraciones de los funcionarios municipales electos. Como balance final, se evidencian las tareas pendientes en materia de transparencia y acceso a la información.

PALABRAS CLAVE. Transparencia, acceso a la información, estudios municipales, administración pública.

\begin{abstract}
Is the culture of transparency rooted in the municipal government? Or is opacity a mean for discretionally managing sits and resources in city halls? In this article, the authors give an account of the state of transparency regarding two basic aspects of municipal government in Mexico: the integration of the council and the remuneration of its members. In theory, the municipal government has been conceived as the space closest to the demands of citizenship, where democratic virtues arise and are exercised. However, what the data shows is that in some city halls of Mexico, there are clear features of arbitrariness and opacity. The authors selected municipalities from the states that held elections in 2017 -Coahuila, Estado de México, Nayarit and Veracruz-, based on the integration and remunerations of the elected municipal officials. The authors analyse the pending agenda in terms of transparency and access to information at the municipal governments.
\end{abstract}

KEY WORDS. Transparency, access to information, municipal studies, public administration, subnational studies.

\title{
I. INTRODUCCIÓN
}

El municipio, como unidad básica de gobierno, encuentra su razón en las teorías del federalismo, que se fundan en las virtudes atribuidas a la división territorial del poder. Se asume que la importancia de la vida municipal radica en las posibilidades que ofrece para la participación social y en la formación del gobierno y su ejercicio (Merino, 1994: 17 y ss.; Guillén, 2016), porque las necesidades y demandas de los ciudadanos se conocen de primera mano. En el federalismo existe una estrecha conexión positiva con la democracia por el autogobierno y la construcción de ciudadanía. Bajo esta óptica, los 
Esta revista forma parte del acervo de la Biblioteca Jurídica Virtual del Instituto de Investigaciones Jurídicas de la UNAM

gobernantes provienen inmediatamente del mismo ámbito que gobiernan y, en principio, los ciudadanos están directamente involucrados en mayor medida con sus autoridades, y más interesados para monitorearlas. Sin transparencia e información sobre su desempeño, esta posibilidad de vigilancia de la sociedad sobre sus gobiernos inmediatos se ve frustrada. Una sociedad que no cuenta con instrumentos ágiles para saber y conocer qué decide su gobierno más cercano y cómo gasta los recursos, tampoco puede construir una relación de confianza con el gobierno en sus distintos niveles, lo cual es esencial para la gobernanza democrática (Merino et al., 2017).

En México, la descentralización de funciones políticas y administrativas también se concibió como uno de los peldaños para avanzar hacia la transición democrática. El federalismo mexicano, soslayado fácticamente en sus dimensiones política, jurídica, administrativa y económica por el presidencialismo hegemónico del PRI, que fue dispositivo de centralización y disciplina, resurgió con las primeras alternancias políticas locales que se sucedieron en 1980 y 1990, las cuales obligaron a voltear la mirada hacia el potencial del ámbito regional como nodo de decisión (Carbonell, 2002; Lujambio, 1995).

A pesar de lo anterior, las virtudes teóricas del municipio no están garantizadas. Como bien apunta María del Carmen Pardo (1994: 280), el municipio puede constituir el motor para el ejercicio democrático, pero también, en ocasiones, supone el freno para este fin. Con frecuencia, en los municipios hay menos capacidades institucionales para ejercer contrapesos al poder, y no existe una masa crítica de organizaciones sociales ni un periodismo independiente que puedan erigirse en los vigilantes de la adecuada acción pública (Ugalde, 2002: 37; Cejudo y Ríos, 2010). Esta labor de vigilancia se ve aún más complicada cuando se carece de información accesible y confiable sobre el ayuntamiento y su desempeño, comenzando por la más básica, que es la relativa a cuántos deben ser los regidores y cuáles son sus percepciones.

A pesar de que las leyes orgánicas municipales en cada estado regulan cómo se componen los ayuntamientos, es decir, cuántos regidores y síndicos habrán de integrarlos, además del presidente municipal, no siempre se acatan dichas disposiciones, ni en cuanto al número de miembros, ni en cuanto a las retribuciones que perciben por su encargo, sino que la variación suele ser la regla. Más aún, a pesar de que la propia Constitución y la Ley General de Transparencia y Acceso a la Información Pública establecen que "las remuneraciones y sus tabuladores serán públicos y deberán especificar y diferenciar la totalidad de sus elementos fijos y variables, tanto en efectivo, como en especie" (CPEUM, artículo 127), y que publi- 
Esta revista forma parte del acervo de la Biblioteca Jurídica Virtual del Instituto de Investigaciones Jurídicas de la UNAM

carlos es una obligación de transparencia (LGTAIP, artículo 70, párrafo VIII), con frecuencia dichos datos no aparecen en los respectivos portales de Internet, ahí donde éstos existen.

A diferencia de la atención que han recibido las remuneraciones de servidores públicos y legisladores en el plano federal, las de los ayuntamientos y su publicidad no han sido un tema recurrente de agenda pública. En los municipios donde hay marcos normativos laxos se abre un amplio margen de manejo discrecional para la integración y las remuneraciones, lo cual, aunque no contraviene los marcos legales, es fuente potencial de conductas arbitrarias y opacas (Roldán, 2013) que sólo están dictadas por voluntad o capricho. La probabilidad de que la discrecionalidad derive en arbitrariedad siempre está latente, en particular si no está garantizado el acceso a la información.

Es por ello que en este estudio se ofrece un panorama del estado de la transparencia municipal respecto de los integrantes del ayuntamiento y de sus remuneraciones. Para lograr este objetivo, se revisaron portales electrónicos y se realizó una serie de solicitudes de información, encontrando una tendencia a la opacidad. Los hallazgos presentados se basan en información ofrecida por los municipios, por lo que, como punta del iceberg, se puede asumir que, bajo la superficie de lo mostrado, yace un cúmulo de casos en los que priva la arbitrariedad y donde la transparencia no ha logrado penetrar.

Para estudiar la transparencia en la integración de los ayuntamientos y las remuneraciones que reciben sus miembros, se eligieron los municipios de cuatro estados: Coahuila, Estado de México, Nayarit y Veracruz. Hay varias razones detrás de esta selección. En primer lugar, son estados que proveen variaciones importantes en términos de población, características geográficas y condiciones socioeconómicas. El Estado de México posee los municipios más grandes en número de habitantes, lo que puede hablar, por un lado, de mayor infraestructura para procesar las solicitudes de información, y de mayor complejidad de gobierno. Esto contrasta con el caso de los municipios de Coahuila y Veracruz, de población media, y con mayor intensidad con Nayarit, cuyos municipios son, en general, pequeños. Las variaciones en los rasgos socioeconómicos, demográficos y de acceso a tecnología dan una idea aproximada de las diversas capacidades institucionales con las que cuentan estos cuatro estados para tener actualizadas las plataformas de transparencia y para facilitar el acceso a la información. Por ejemplo, mientras en Coahuila el 52.2\% de los hogares cuenta con acceso a Internet, en Veracruz esto sólo sucede para el $30.3 \%$, en el Estado de México para el $45.3 \%$ y en Nayarit para el $46 \%$ 
Esta revista forma parte del acervo de la Biblioteca Jurídica Virtual del Instituto de Investigaciones Jurídicas de la UNAM

(INEGI, 2016). Estas diferencias también hablan de distintos niveles en capacidad de vigilancia social; es decir, los cuatro estados condensan e ilustran la gran variabilidad municipal. Así, sin que se pretenda presentar una muestra representativa, los casos elegidos nos permiten ofrecer una selección muestral de la variabilidad que existe en el país. Una razón más por la que se decidió centrar este estudio en los estados mencionados es porque los cuatro tuvieron un proceso electoral durante 2017, por lo que se esperaría encontrar una dinámica más intensa en el monitoreo del manejo de los recursos públicos.

\section{El marco Normativo PARA LA INTEGRACión DE LOS MUNICIPIOS Y LA REMUNERACIÓN DE LOS INTEGRANTES}

En México, los congresos locales tienen la facultad de establecer la forma de combinar los principios de representación de mayoría relativa (MR) y proporcional (RP) en la conformación de sus ayuntamientos; sin embargo, en 2013 la Suprema Corte de Justicia de la Nación (SCJN) estableció que el parámetro de integración de la Cámara de Diputados federal (60\% por MR y 40\% por RP) se aplicaría en el ámbito municipal (Tesis P./J. 19/2013). Debido a que gran parte de las decisiones del cabildo se toman por mayoría simple, por ejemplo, la aprobación de un reglamento o una política pública (Inafed, 2012: 41), es importante garantizar los contrapesos por vía de una suficiente RP. ${ }^{1}$

Por lo anterior, es fundamental conocer los datos básicos del gobierno municipal: ¿cuántos funcionarios lo integran?, ¿cómo fueron elegidos?, ¿cuáles son sus remuneraciones?, y, sobre todo, si la integración y los salarios están apegados a la ley. Para acercarnos a estos datos, en este artículo empleamos las herramientas de transparencia y acceso a la información pública.

Dado que una de las obligaciones básicas de todo órgano de gobierno es la publicación de su directorio de servidores públicos, organigrama, facultades y atribuciones (LGTAIP, artículo 70, fracciones II y VII), esta información debería estar disponible en la página de Internet propia de

1 Sólo ciertas resoluciones, como la relativa al patrimonio inmobiliario municipal; a actos o convenios que comprometan al municipio por un plazo mayor al periodo de duración de la administración municipal; o a una función o servicio municipal que involucre al gobierno estatal, deberá votarse por mayoría calificada (2/3 partes de los integrantes del cabildo). Esta modificación se introdujo con la reforma del artículo 115 constitucional de 1999. 
Esta revista forma parte del acervo de la Biblioteca Jurídica Virtual del Instituto de Investigaciones Jurídicas de la UNAM

cada municipio. ${ }^{2}$ Ello nos permitiría contrastar la integración real con lo que establecen los marcos normativos.

De los 395 municipios que integran el universo de estudio de este trabajo, se encontró que 316 municipios (80\%) sí tienen una página de Internet, mientras que 79 municipios no la tienen (20\%). ${ }^{3}$ De estos municipios sin página de Internet, el 96.2\% (76 municipios) se encuentra en el estado de Veracruz. ${ }^{4}$

Los municipios que no tienen una página de Internet son pequeños en población: el 81\% (64 municipios) tiene menos de 30 mil habitantes. A medida que los municipios crecen en población, se esperaría que contaran con mayores recursos y capacidades institucionales para cumplir la obligación de transparencia de tener una página de Internet.

En los siguientes cuadros se muestra el panorama inicial sobre la posibilidad de acceder a la información de interés desde las páginas de Internet de los municipios que son objeto de este estudio. En el primero se da cuenta de los municipios que poseen página electrónica propia.

\section{Cuadro 1. Existencia de páginas de Internet MUNICIPALES POR ESTADO}

\begin{tabular}{|l|c|c|}
\hline \multicolumn{1}{|c|}{ Municipios de: } & Con página de Internet & Sin página de Internet \\
\hline Coahuila (38) & $36(94.7 \%)$ & $2(5.3 \%)$ \\
\hline Estado de México (125) & $125(100 \%)$ & 0 \\
\hline Nayarit (20) & $19(95 \%)$ & $1(5 \%)$ \\
\hline Veracruz (212) & $136(64.1 \%)$ & $76(35.9 \%)$ \\
\hline Total & $316(80 \%)$ & $79(20 \%)$ \\
\hline
\end{tabular}

FuENTE: elaboración propia con base en la búsqueda y revisión de páginas de Internet de los municipios de los estados de Coahuila, México, Nayarit y Veracruz.

2 El artículo décimo transitorio de la LGTAIP (2015) estableció que los municipios con población menor a 70 mil habitantes cumplirán con las obligaciones de transparencia de conformidad con sus posibilidades presupuestarias y, de forma subsidiaria, podrán solicitar al organismo garante local la divulgación vía Internet de la información de dichas obligaciones.

3 La información sobre los directorios de servidores públicos de los municipios sin página de Internet se consultó en la base de datos del Instituto Nacional para el Federalismo y el Desarrollo Municipal (Inafed), disponible en: www.snim.rami.gob.mx.

4 El periodo de revisión de las páginas de Internet de los ayuntamientos se realizó de septiembre a noviembre de 2016. 
Esta revista forma parte del acervo de la Biblioteca Jurídica Virtual del Instituto de Investigaciones Jurídicas de la UNAM

Sin embargo, poseer un portal electrónico no es sinónimo de facilitar acceso a la información. De aquellos municipios que sí cuentan con página de Internet, la disponibilidad de la información es variable, destacando negativamente el caso de Veracruz, donde la mayoría de los portales carece de información respecto de remuneraciones, aunque sí se despliega el directorio de los integrantes del cabildo.

\section{Cuadro 2. Disponibilidad de información} EN LAS PÁGINAS DE INTERNET DE LOS MUNICIPIOS

\begin{tabular}{|l|c|c|c|}
\hline \multicolumn{1}{|c|}{ Municipios de: } & $\begin{array}{c}\text { Información } \\
\text { completa }\end{array}$ & $\begin{array}{c}\text { Información } \\
\text { incompleta }\end{array}$ & Sin información \\
\hline Coahuila (38) & $28(73.6 \%)$ & $9(23 \%)$ & $1(2.6 \%)$ \\
\hline $\begin{array}{l}\text { Estado de México } \\
(125)\end{array}$ & $75(60 \%)$ & $37(29.6 \%)$ & $13(10.4 \%)$ \\
\hline Nayarit (19) & $9(47.3 \%)$ & $10(52.7 \%)$ & 0 \\
\hline Veracruz (136) & $43(31.6 \%)$ & $8(5.9 \%)$ & $85(62.5 \%)$ \\
\hline
\end{tabular}

Fuente: elaboración propia con base en la búsqueda y revisión de páginas de Internet de los municipios de los estados de Coahuila, México, Nayarit y Veracruz.

Por lo anterior, fue necesario realizar solicitudes para acceder a la información de nuestro interés. No obstante, los municipios que peor desempeño mostraron en el despliegue de la información en sus portales también tuvieron fallas operativas en sus plataformas de acceso, las cuales hicieron imposible el trámite de la solicitud.

\section{Cuadro 3. Solicitudes de acceso a la información (SEPTIEMBRE-OCTUBRE DE 2OI6)}

\begin{tabular}{|l|c|c|c|}
\hline \multicolumn{1}{|c|}{ Municipios de: } & $\begin{array}{c}\text { Número de } \\
\text { solicitudes }\end{array}$ & $\begin{array}{c}\text { Con respuesta } \\
\text { (completa) }\end{array}$ & Sin respuesta \\
\hline Coahuila & 12 & 9 & 3 \\
\hline Estado de México & 50 & 36 & 14 \\
\hline Nayarit & 1 & 0 & 1 \\
\hline Veracruz $^{*}$ & 0 & - & - \\
\hline
\end{tabular}

FuENTE: elaboración propia con base en el envío de solicitudes de acceso a la información a través de los sistemas Infomex de los estados de Coahuila, México y Nayarit.

${ }^{*}$ No fue posible enviar solicitudes de acceso a los municipios de Veracruz debido a las fallas en la plataforma nacional de transparencia y en el sistema Infomex local. 
Esta revista forma parte del acervo de la Biblioteca Jurídica Virtual del Instituto de Investigaciones Jurídicas de la UNAM http://www.juridicas.unam.mx/ https://biblio.juridicas.unam.mx/bjv

\section{LA INTEGRACIÓN DE LOS AYUNTAMIENTOS}

A fin de iniciar el análisis de la integración y las remuneraciones de los gobiernos municipales, vale la pena retomar los principales datos sociodemográficos de los estados a estudiar. Como se mencionó, se trata de cuatro casos muy contrastantes tanto en la dimensión territorial como en el número de municipios. También se diferencian las condiciones socioeconómicas de los estados, destacando negativamente el caso de Veracruz, donde el $62 \%$ de la población se encuentra en situación de pobreza.

\section{Cuadro 4. Datos sociodemográficos de los estados}

\begin{tabular}{|l|c|c|c|c|c|c|c|}
\hline $\begin{array}{c}\text { Municipios } \\
\text { de: }\end{array}$ & Población & $\begin{array}{c}\text { Superficie } \\
\text { en } \mathrm{km}^{2}\end{array}$ & $\begin{array}{c}\text { Muni- } \\
\text { cipios }\end{array}$ & $\begin{array}{c}\text { PIB de la } \\
\text { entidad } \\
\text { (millones } \\
\text { de pesos) }\end{array}$ & $\begin{array}{c}\% \text { po- } \\
\text { blación } \\
\text { en po- } \\
\text { breza }\end{array}$ & $\begin{array}{c}\text { Sin- } \\
\text { dicos }\end{array}$ & $\begin{array}{l}\text { Regi- } \\
\text { dores }\end{array}$ \\
\hline Coahuila & $2,954,915$ & 151,595 & 38 & 464,445 & 30.02 & 76 & 396 \\
\hline $\begin{array}{l}\text { Estado de } \\
\text { México }\end{array}$ & $16,187,608$ & 22,351 & 125 & $1,593,572$ & 47.9 & 139 & 1343 \\
\hline Nayarit & $1,181,050$ & 27,857 & 20 & 92,079 & 40.5 & 20 & 197 \\
\hline Veracruz & $8,112,505$ & 71,826 & 212 & 854,065 & 62.2 & 212 & 629 \\
\hline
\end{tabular}

Fuente: elaboración propia con base en Coneval (2016), Inafed (2016) e INEGI (2015a).

En buena lógica federal, se entiende que la diversidad de los municipios en cuanto a población, electores, condiciones económicas y sociales, etcétera, justifica que exista una variación en los diseños institucionales de los estados sobre los criterios para determinar el número de los integrantes de sus ayuntamientos. Por esta razón, la Constitución Política faculta a los congresos estatales para establecer dichos criterios en su Constitución local y en su ley orgánica municipal (Inafed, 2012: 24), y, en algunos casos, también en su ley electoral local.

Existen tres grandes criterios para la definición del número de integrantes de cada cabildo: 1) a partir de la población de cada municipio; 2) el número de electores, y 3) el diferencial, es decir, desde la propia ley orgánica municipal se establecen criterios para la conformación de los ayuntamientos de la entidad (Hurtado, 2012: 37).5 El Estado de México y

515 estados implementan el criterio poblacional; 12 estados utilizan el criterio diferencial; 2 (Coahuila y Nayarit) recurren al criterio de número de electores, y los 2 estados 
Esta revista forma parte del acervo de la Biblioteca Jurídica Virtual del Instituto de Investigaciones Jurídicas de la UNAM http://www.juridicas.unam.mx/ https://biblio.juridicas.unam.mx/bjv https://revistas.juridicas.unam.mx/

DOI: http://dx.doi.org/10.22201/iij.25940082e.2018.6.12464

TRANSPARENCIA EN LOS AYUNTAMIENTOS: DISCRECIONALIDAD EN SU INTEGRACIÓN...

Veracruz utilizan el criterio de población, mientras que Coahuila y Nayarit toman el criterio de electores.

\section{Cuadro 5. Número de integrantes de los ayuntamientos}

\begin{tabular}{|c|c|c|c|c|c|}
\hline \multirow{2}{*}{$\begin{array}{l}\text { Municipios } \\
\text { de: }\end{array}$} & \multirow{2}{*}{ Rangos (habitantes) } & \multicolumn{2}{|c|}{ Sindicos } & \multicolumn{2}{|c|}{ Regidores } \\
\hline & & $M R$ & $R P$ & $M R$ & $R P$ \\
\hline \multirow{5}{*}{$\begin{array}{l}\text { Estado de } \\
\text { México }\end{array}$} & $\begin{array}{l}\text { I. Menos de } 150 \\
\text { mil }\end{array}$ & 1 & 0 & $6(60 \%)$ & $4(40 \%)$ \\
\hline & $\begin{array}{l}\text { II. Más de } 150 \mathrm{mil} \\
\text { y menos de } 500 \mathrm{mil}\end{array}$ & 1 & 0 & $7(53.80 \%)$ & $6(46.20 \%)$ \\
\hline & $\begin{array}{l}\text { III. Más de } 500 \text { mil } \\
\text { habitantes y menos } \\
\text { de } 1 \text { millón }\end{array}$ & 2 & 1 & $9(56.25 \%)$ & $7(43.75 \%)$ \\
\hline & $\begin{array}{l}\text { IV. Más de } 1 \text { millón } \\
\text { de habitantes }\end{array}$ & 2 & 1 & $11(57.90 \%)$ & $8(42.10 \%)$ \\
\hline & Rangos (habitantes) & \multicolumn{2}{|c|}{ Síndicos $(M R)$} & \multicolumn{2}{|c|}{ Regidores $(R P)$} \\
\hline \multirow{8}{*}{ Veracruz } & I. Hasta $40 \mathrm{mil}$ & \multicolumn{2}{|c|}{1} & \multicolumn{2}{|c|}{1} \\
\hline & $\begin{array}{l}\text { II. Más de } 40 \text { mil y } \\
\text { hasta } 70 \text { mil }\end{array}$ & \multicolumn{2}{|c|}{1} & \multicolumn{2}{|c|}{3} \\
\hline & $\begin{array}{l}\text { III. Más de } 70 \mathrm{mil} \\
\text { y hasta } 125 \mathrm{mil}\end{array}$ & \multicolumn{2}{|c|}{1} & \multicolumn{2}{|c|}{5} \\
\hline & $\begin{array}{l}\text { IV. Más de } 125 \mathrm{mil} \\
\text { y hasta } 250 \mathrm{mil}\end{array}$ & \multicolumn{2}{|c|}{1} & \multicolumn{2}{|c|}{7} \\
\hline & $\begin{array}{l}\text { V. Más de } 250 \text { mil y } \\
\text { hasta } 400 \text { mil }\end{array}$ & \multicolumn{2}{|c|}{1} & \multicolumn{2}{|c|}{11} \\
\hline & VI. Más de 400 mil & \multicolumn{2}{|c|}{1} & \multicolumn{2}{|c|}{13} \\
\hline & \multirow[b]{2}{*}{ Rangos (electores) } & \multicolumn{2}{|c|}{ Sindicos } & \multicolumn{2}{|c|}{ Regidores } \\
\hline & & $M R$ & $\begin{array}{l}\text { la. } \\
\text { Min. }\end{array}$ & $M R$ & $R P$ \\
\hline \multirow{4}{*}{ Coahuila } & I. Hasta $15 \mathrm{mil}$ & 1 & 1 & $5(71.40 \%)$ & $2(28.60 \%)$ \\
\hline & $\begin{array}{l}\text { II. De 15,001 hasta } \\
40 \mathrm{mil}\end{array}$ & 1 & 1 & $7(63.60 \%)$ & $4(36.40 \%)$ \\
\hline & $\begin{array}{l}\text { III. De } 40,001 \text { has- } \\
\text { ta } 80 \text { mil }\end{array}$ & 1 & 1 & $9(60 \%)$ & $6(40 \%)$ \\
\hline & IV. Más de 80,001 & 1 & 1 & $11(64.70 \%)$ & $6(35.30 \%)$ \\
\hline
\end{tabular}

restantes (Aguascalientes y Tabasco) combinan algunos de los criterios anteriores (Hurtado, 2012: 38). 
Esta revista forma parte del acervo de la Biblioteca Jurídica Virtual del Instituto de Investigaciones Jurídicas de la UNAM http://www.juridicas.unam.mx/ https://biblio.juridicas.unam.mx/bjv

\begin{tabular}{|c|c|c|c|c|}
\hline & \multirow{2}{*}{ Rangos (electores) } & \multirow{2}{*}{ Sindicos $(M R)$} & \multicolumn{2}{|c|}{ Regidores } \\
\hline & & & $M R$ & $\mathrm{RP}$ \\
\hline \multirow{4}{*}{ Nayarit } & I. Hasta 15 mil & 1 & $5(71.40 \%)$ & $2(28.60 \%)$ \\
\hline & $\begin{array}{l}\text { II. De } 15 \text { mil hasta } \\
40 \text { mil }\end{array}$ & 1 & $7(70 \%)$ & $3(30 \%)$ \\
\hline & $\begin{array}{l}\text { III. De } 40 \text { mil hasta } \\
80 \mathrm{mil}\end{array}$ & 1 & $9(69.20 \%)$ & $4(30.80 \%)$ \\
\hline & IV. Más de 80 mil & 1 & $11(68.75 \%)$ & $5(31.25 \%)$ \\
\hline
\end{tabular}

FUENTE: elaboración propia con base en CEEC (artículo 19), LEEN (artículo 23), LOMEM (artículo 16), CEEV (artículo 16) y LOMLEV (artículo 21).

Llama la atención que en los estados más grandes, Estado de México y Veracruz, los rangos de población para determinar el número de integrantes de los ayuntamientos son muy amplios, y, por lo tanto, existe el riesgo de que se genere una sobre o sub-representación. A manera de ejemplo, en el Estado de México el primer rango establece que todos los municipios que poseen hasta 150 mil habitantes tendrán 12 ediles, incluyendo al presidente municipal. Así, este criterio determina que, dentro de los 14 municipios que entran en este rango, haya municipios pequeños, como Zacazonapan, que, con poco más de 4 mil habitantes, tiene el mismo número de ediles que Almoloya de Juárez, el cual tiene más de 147 mil 653. Algo similar sucede para Veracruz, en donde los municipios de Landero y Aquila, con menos de 2 mil habitantes, tienen el mismo número de ediles (3) que Jáltipan, que posee casi 50 mil habitantes.

En lo que respecta a la distribución de los cargos, según los principios de MR y RP, lo que persiste es una altísima heterogeneidad, a pesar de los criterios jurisprudenciales mencionados, no sólo en la integración real de los municipios, sino incluso en el propio marco normativo estatal.

Con excepción del Estado de México, las fórmulas de integración de los ayuntamientos establecidas en las normas estatales exceden el porcentaje de $60 \%$ para regidores por el principio de MR, con lo que se asegura la prevalencia de la fuerza política mayoritaria en el municipio. Los municipios que de manera más acentuada exceden el parámetro de $60 \%$ son los más pequeños en población. Por ejemplo, Coahuila establece $71.4 \%$ de MR para municipios con menos de 150 mil electores, lo que equivale a 19 municipios de un total de 38, es decir, la mitad de los municipios. Quizá el caso más notorio es el de Nayarit, en donde la norma establece más del $60 \%$ para asignar regidores por MR. Por el contrario, cabe destacar que en el Estado de México la norma establece que todos los municipios se 
Esta revista forma parte del acervo de la Biblioteca Jurídica Virtual del Instituto de Investigaciones Jurídicas de la UNAM

ciñan al criterio de la SCJN, respetando la proporción 60/40, e incluso a medida que los municipios son más grandes, otorgan un número mayor de regidores de RP.

En Nayarit, Coahuila y el Estado de México existe un tope a la representación del partido que haya ganado por el principio de MR, el cual le impide participar en el reparto de integrantes del ayuntamiento por RP. El diseño institucional para la elección de los regidores de los ayuntamientos de Veracruz es singular, ya que todos ellos se eligen por el principio de RP, es decir, participa en dicho reparto el partido que recibió más votos en la elección de presidente municipal (CEEV, artículo 238).

De la norma a los hechos: diferencias en la integración de los municipios

El primer aspecto que interesa analizar es la correspondencia en la integración numérica de los municipios, es decir, si el número total de ediles se apega a lo que establecen las normas locales.

La información pública muestra que los municipios de Coahuila, México y Nayarit cumplen con el número de integrantes de sus ayuntamientos, según lo mandatado en sus respectivas leyes. Las irregularidades que se encontraron en la integración de los ayuntamientos de Veracruz fueron: de un total de 212 municipios, en 97 (45.75\%) de ellos el número de regidores y síndicos es superior al establecido en su ley municipal ( 95 rebasan el número de regidores y 2 el de síndicos). El incumplimiento es mayor en los municipios con población superior a los 30 mil habitantes, pues de 64 municipios en este rango, $52(81.25 \%)$ tienen ayuntamientos con un número superior de integrantes a lo establecido en la ley.

Respecto de la figura de síndicos, únicamente dos de los municipios analizados exceden el número contemplado en la norma. Nuevamente, ambos se encuentran en Veracruz: Chinameca e Hidalgotitlán. El primero gobernado por la coalición PRI-PVEM, y el segundo, por el partido Alternativa Veracruzana (AVE).

Una posible razón detrás de que los municipios de Veracruz recurrentemente excedan el número de ediles establecidos normativamente es que la Ley Orgánica municipal del estado (LOMLEV, artículo 21) faculta al congreso local para hacer modificaciones en el número de integrantes de los ayuntamientos con base en el censo de población. En cualquier caso, la norma no señala de manera clara cómo debe incrementarse el número de ediles, lo que implica un acto arbitrario que, según lo definido por 
Esta revista forma parte del acervo de la Biblioteca Jurídica Virtual del Instituto de Investigaciones Jurídicas de la UNAM

Fraga (1987: 232), "tiene lugar cuando la ley deja a la administración un poder libre de apreciación para decidir si debe obrar o abstenerse o en qué momento debe obrar o cómo debe obrar o en fin qué contenidos va a dar a su actuación”. Incrementar a discreción el número de ediles en un ayuntamiento sólo puede servir para que el presidente municipal tenga un mayor número de asientos a distribuir y, con ello, un margen más amplio para negociar con los grupos políticos existentes.

Un aspecto llamativo es que en el $76 \%$ de los municipios que rebasan el número de ediles establecidos en la ley, fue imposible tener datos sobre las remuneraciones de éstos, lo que lleva a pensar que la arbitrariedad y la opacidad caminan de la mano.

En segundo lugar, en lo que respecta a la asignación de cargos por los principios de MR y RP, Nayarit y el Estado de México se apegan a lo que establecen sus respectivos códigos electorales, aunque, como se señaló más arriba, esta disposición normativa establece una sobrerrepresentación del principio de mayoría. Así, en promedio, los municipios de Nayarit cuentan con un 69\% del total de regidores nombrados por el principio de MR y $31 \%$ por el principio de RP. En el Estado de México, en promedio, el 58\% de regidores de cada ayuntamiento llegó a su cargo por el principio de MR y el $42 \%$ por el principio de RP.

Ahora bien, en lo que respecta a Coahuila, sólo el $60 \%$ de sus municipios se apega a lo establecido en el Código Electoral estatal, excediendo el número de regidores que debería haberse nombrado por MR. En promedio, los ayuntamientos de este estado se componen por un $67 \%$ de regidores electos por MR y 33\% electos por RP.

Por las particularidades de Veracruz, donde no existen regidores por MR, este estado queda fuera de este análisis.

\section{LAS REMUNERACIONES EN LOS AYUNTAMIENTOS}

En la Constitución se señala que "los servidores públicos de la Federación, de las entidades federativas, de los Municipios y de las demarcaciones territoriales de la Ciudad de México... recibirán una remuneración adecuada e irrenunciable por el desempeño de su función, empleo, cargo o comisión, que deberá ser proporcional a sus responsabilidades" (CPEUM, artículo 127). Las bases constitucionales de las remuneraciones establecen que ningún servidor público ganará más que el presidente de la República ni deberá ganar igual o más que su superior jerárquico; que no se cubrirán jubilacio- 
Esta revista forma parte del acervo de la Biblioteca Jurídica Virtual del Instituto de Investigaciones Jurídicas de la UNAM

nes, pensiones, o liquidaciones que no estén en ley, y que los tabuladores de las remuneraciones serán públicos.

Este marco normativo, vigente desde 2009, tiene por objeto reducir la discrecionalidad y la arbitrariedad en el ejercicio del gasto público en materia de remuneraciones. Las Constituciones del Estado de México (CPELSM, artículo 147) y Veracruz (CPEV, artículo 82) incorporan en su texto todas las bases en materia de remuneraciones establecidas en la carta magna. Incluso, en el Estado de México existe el Consejo Consultivo de Valoración Salarial, órgano auxiliar del Poder Legislativo, que anualmente formula recomendaciones para instrumentar las disposiciones normativas de las remuneraciones de los servidores públicos de los municipios; ${ }^{6} \sin$ embargo, es potestad de los ayuntamientos acatarlas o no.

En la Constitución de Coahuila sólo se incorpora el concepto de "remuneración adecuada" de los servidores públicos, y es determinada anualmente en el presupuesto de egresos correspondiente (CPCZ, artículo 187). En Nayarit se establece que "la ley determinará los conceptos de retribuciones y límites salariales de los servidores públicos, que no podrá ser igual o mayor que su superior jerárquico..." (CPELSN, artículo 137). Es decir, tanto Coahuila como Nayarit dejan grandes márgenes de discrecionalidad para ajustarse al modelo establecido en la Constitución. Aunque en la reforma constitucional de agosto de 2009 se estableció la obligación de emitir una ley reglamentaria en la materia, ninguno de los estados estudiados cuenta con ella.

A pesar de la falta de esta legislación secundaria, la Ley General de Transparencia y Acceso a la Información Pública señala que será pública la información sobre remuneración de los servidores públicos, incluyendo sueldos, prestaciones, gratificaciones, primas y otras compensaciones (LGTAIP, artículo 70, fracción VIII). Este modelo es el que siguen las leyes de transparencia locales.

De los 395 municipios que abarca este estudio, sólo se tuvo acceso a la información de las remuneraciones mensuales de 222 a través de sus propias páginas o de las 63 solicitudes de acceso a la información realizadas; es decir, sólo se pudo integrar información sobre remuneraciones para el $56.2 \%$ de los casos, a pesar de que es un dato de obligaciones de transparencia.

6 Recomendaciones publicadas en el Periódico Oficial del Estado de México el 25 de febrero de 2016, disponible en: http://legislacion.edomex.gob.mx/sites/legislacion.edomex. gob.mx/files/files/pdf/gct/2016/feb255.pdf. 
Esta revista forma parte del acervo de la Biblioteca Jurídica Virtual del Instituto de Investigaciones Jurídicas de la UNAM http://www.juridicas.unam.mx/ https://biblio.juridicas.unam.mx/bjv https://revistas.juridicas.unam.mx/

DOI: http://dx.doi.org/10.22201/iij.25940082e.2018.6.12464

PESCHARD MARISCAL / SALAZAR REBOLLEDO / OLEA GÓMEZ

\section{Cuadro 6. Acceso a la información en materia DE REMUNERACIONES POR ESTADO}

\begin{tabular}{|l|c|c|}
\hline \multicolumn{1}{|c|}{ Municipios de: } & \multicolumn{2}{c|}{$\begin{array}{c}\text { Número de municipios con información } \\
\text { disponible (portales y solicitudes) }\end{array}$} \\
\hline Coahuila & 35 de 38 & $92.10 \%$ \\
\hline Estado de México & 117 de 125 & $93.60 \%$ \\
\hline Nayarit & 19 de 20 & $95 \%$ \\
\hline Veracruz & 51 de 212 & $24 \%$ \\
\hline
\end{tabular}

- Fuente: consulta de las páginas de Internet de los municipios en sus secciones de trans- parencia y solicitudes de acceso.

\section{Gráfica 1. Remuneración Promedio de los munícipes POR CARGO Y ESTADO}

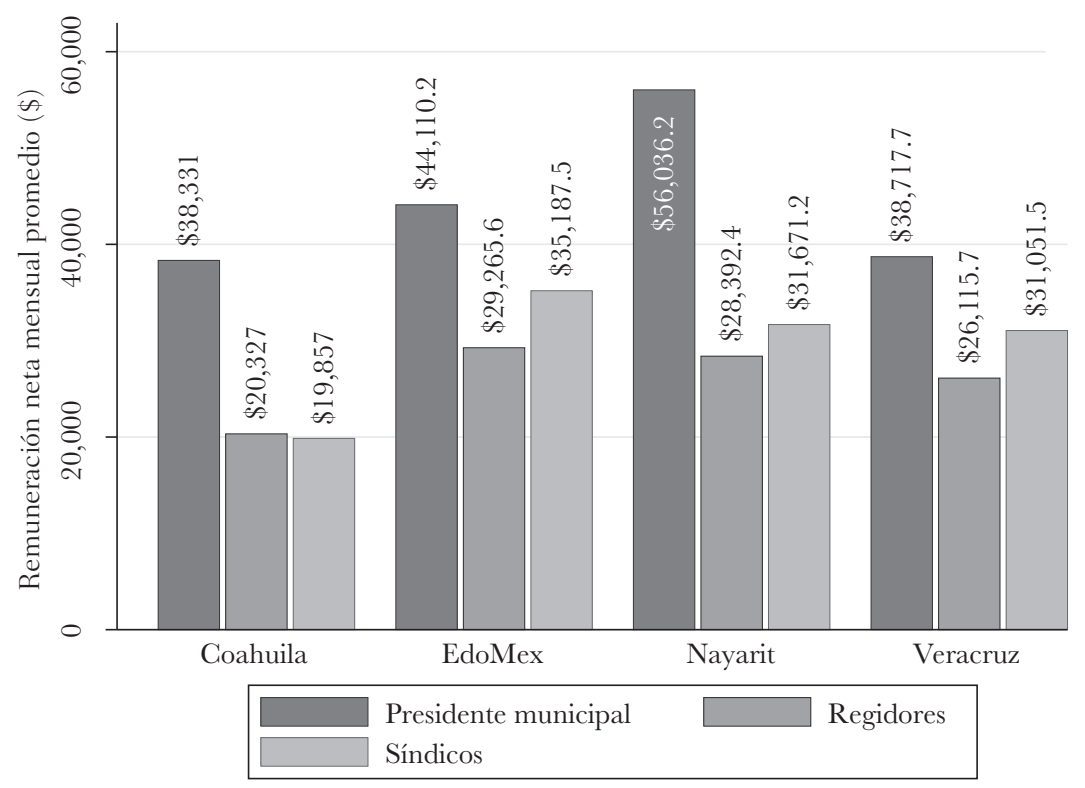

Fuente: elaboración propia con base en las páginas de Internet de los municipios en sus secciones de transparencia, según la disponibilidad de información señalada en el cuadro 6.

Una de las consecuencias más graves de la arbitrariedad en la integración de los ayuntamientos es su traducción en términos monetarios (gráfica 1). La equivalencia de dichas remuneraciones en salarios mínimos vigentes (80.04 pesos diarios) es, en promedio, de 19.4 salarios mínimos para presidente municipal, 14.3 para síndicos y 12.2 para regidores. 
Esta revista forma parte del acervo de la Biblioteca Jurídica Virtual del Instituto de Investigaciones Jurídicas de la UNAM

Dado que la norma no presenta criterios específicos para evaluar si las remuneraciones se apegan a la ley, cabe preguntarse ¿de qué dependen los salarios que perciben los ediles? Una explicación inicial sería que municipios de mayor población son más complejos de gobernar, y, por ello, el tamaño de los municipios podría estar relacionado con los salarios. Sin embargo, los datos expuestos en el cuadro 7 muestran que las variaciones de las remuneraciones de cualquiera de los integrantes de los ayuntamientos no dependen de la mayor o menor población del municipio. En ningún estado se puede apreciar que una mayor población lleve a mayores remuneraciones. Incluso en Nayarit, los puestos de síndico y regidor están mejor pagados en los municipios pequeños (con menos de 70 mil habitantes) que en los grandes. En realidad, el municipio que presenta mayor nivel salarial para el presidente municipal (Progreso, Coahuila) es también uno de los que tiene la menor población (3,473 habitantes).

\section{Cuadro 7. Remuneraciones mensuales netas promedio}

DE LOS INTEGRANTES DE LOS AYUNTAMIENTOS, POR RANGOS DE POBLACIÓN

\begin{tabular}{|c|c|c|c|c|}
\hline $\begin{array}{l}\text { Puesto: presidente } \\
\text { municipal }\end{array}$ & Coahuila & $\begin{array}{l}\text { Estado de } \\
\text { México }\end{array}$ & Nayarit & Veracruz \\
\hline $\begin{array}{l}\text { I. Menos de } 70 \text { mil ha- } \\
\text { bitantes }\end{array}$ & $36,057.07$ & $40,737.91$ & $54,488.96$ & $35,199.55$ \\
\hline $\begin{array}{l}\text { II. Entre } 70 \text { mil y } 150 \\
\text { mil habitantes }\end{array}$ & $40,189.79$ & $51,678.51$ & $70,396.38$ & $39,697.26$ \\
\hline $\begin{array}{l}\text { III. Entre } 150 \text { mil y } 300 \\
\text { mil habitantes }\end{array}$ & $36,430.64$ & $62,250.06$ & $35,942.00$ & $64,410.82$ \\
\hline $\begin{array}{l}\text { IV. Entre } 300 \text { mil y } 500 \\
\text { mil habitantes }\end{array}$ & $66,491.70$ & $56,999.71$ & - & $45,000.00$ \\
\hline $\begin{array}{l}\text { VI. Entre } 500 \text { mil y } 1 \\
\text { millón de habitantes }\end{array}$ & $75,828.40$ & $73,672.13$ & - & - \\
\hline $\begin{array}{l}\text { VII. Más de } 1 \text { millón de } \\
\text { habitantes }\end{array}$ & - & $54,024.62$ & - & - \\
\hline \multicolumn{5}{|l|}{ Puesto: síndico } \\
\hline $\begin{array}{l}\text { I. Menos de } 70 \text { mil ha- } \\
\text { bitantes }\end{array}$ & $17,132.28$ & $30,388.73$ & $32,299.68$ & $26,638.96$ \\
\hline $\begin{array}{l}\text { II. Entre } 70 \text { mil y } 150 \\
\text { mil habitantes }\end{array}$ & $23,936.78$ & $43,641.84$ & $23,308.00$ & $31,829.10$ \\
\hline $\begin{array}{l}\text { III. Entre } 150 \text { mil y } 300 \\
\text { mil habitantes }\end{array}$ & $19,260.55$ & $52,378.74$ & $27,695.00$ & $61,631.58$ \\
\hline
\end{tabular}


Esta revista forma parte del acervo de la Biblioteca Jurídica Virtual del Instituto de Investigaciones Jurídicas de la UNAM http://www.juridicas.unam.mx/ https://biblio.juridicas.unam.mx/bjv

DOI: http://dx.doi.org/10.22201/iij.25940082e.2018.6.12464

PESCHARD MARISCAL / SALAZAR REBOLLEDO / OLEA GÓMEZ

\begin{tabular}{|c|c|c|c|c|}
\hline $\begin{array}{l}\text { IV. Entre } 300 \text { mil y } 500 \\
\text { mil habitantes }\end{array}$ & $37,340.08$ & $55,081.87$ & - & $45,000.00$ \\
\hline $\begin{array}{l}\text { VI. Entre } 500 \text { mil y } 1 \\
\text { millón de habitantes }\end{array}$ & $48,301.39$ & $66,079.39$ & - & - \\
\hline $\begin{array}{l}\text { VII. Más de } 1 \text { millón de } \\
\text { habitantes }\end{array}$ & - & $44,533.94$ & - & - \\
\hline \multicolumn{5}{|l|}{ Puesto: regidor } \\
\hline $\begin{array}{l}\text { I. Menos de } 70 \text { mil ha- } \\
\text { bitantes }\end{array}$ & $15,898.58$ & $23,749.90$ & $28,632.40$ & $20,948.50$ \\
\hline $\begin{array}{l}\text { II. Entre } 70 \text { mil y } 150 \\
\text { mil habitantes }\end{array}$ & $23,936.78$ & $38,730.37$ & $24,775.96$ & $29,288.41$ \\
\hline $\begin{array}{l}\text { III. Entre } 150 \text { mil y } 300 \\
\text { mil habitantes }\end{array}$ & $19,260.55$ & $42,350.38$ & $26,060.00$ & $57,437.36$ \\
\hline $\begin{array}{l}\text { IV. Entre } 300 \text { mil y } 500 \\
\text { mil habitantes }\end{array}$ & $37,340.08$ & $48,238.16$ & - & $45,000.00$ \\
\hline $\begin{array}{l}\text { VI. Entre } 500 \text { mil y } 1 \\
\text { millón de habitantes }\end{array}$ & $48,301.39$ & $56,968.82$ & - & - \\
\hline $\begin{array}{l}\text { VII. Más de } 1 \text { millón de } \\
\text { habitantes }\end{array}$ & - & $39,270.90$ & - & - \\
\hline
\end{tabular}

FueNTE: elaboración propia con base en las páginas de Internet de los municipios en sus secciones de transparencia, según la disponibilidad de información señalada en el cuadro 6.

Si bien son las legislaturas de los estados las que tienen la facultad de aprobar las leyes de ingresos de los municipios, éstos "administran libremente su hacienda" y aprueban su presupuesto de egresos, el cual debe contener los tabuladores de las remuneraciones de los servidores públicos municipales (CPEUM, artículo 115, fracción IV); es decir, no hay consistencia entre la norma y las atribuciones de los municipios. Los escándalos por las altas remuneraciones que reciben los presidentes municipales se enmarcan en esta contradicción entre las leyes estatales y las facultades de los municipios libres. Un ejemplo de lo anterior fue la nota que en 2011 publicó la revista Proceso sobre el salario que recibía el alcalde del municipio de Tlalnepantla en el Estado de México, la cual ascendía a 749 mil pesos como remuneración bruta, ganando más que el presidente de la República o el gobernador del estado (Pérez, 2011).

Los diez municipios que presentan mayor remuneración para quienes encabezan sus ayuntamientos son los siguientes: 
Esta revista forma parte del acervo de la Biblioteca Jurídica Virtual del Instituto de Investigaciones Jurídicas de la UNAM http://www.juridicas.unam.mx/ https://biblio.juridicas.unam.mx/bjv https://revistas.juridicas.unam.mx/

DOI: http://dx.doi.org/10.22201/iij.25940082e.2018.6.12464

TRANSPARENCIA EN LOS AYUNTAMIENTOS: DISCRECIONALIDAD EN SU INTEGRACIÓN...

Cuadro 8. Ranking de Remuneraciones

PARA PRESIDENTE MUNICIPAL

\begin{tabular}{|c|l|l|c|c|c|}
\hline No. & Estado & \multicolumn{1}{|c|}{ Municipio } & $\begin{array}{c}\text { Salario neto en } \\
\text { pesos }\end{array}$ & $\begin{array}{c}\text { Equivalencia } \\
\text { en salarios } \\
\text { mínimos }\end{array}$ & $\begin{array}{c}\text { Partido } \\
\text { político }\end{array}$ \\
\hline 1 & Coahuila & Progreso & $138,287.33$ & 62.28 & PRI \\
\hline 2 & Nayarit & Xalisco & $121,366.64$ & 54.66 & PAN \\
\hline 3 & Nayarit & Del Nayar & $120,720.34$ & 54.37 & PRI \\
\hline 4 & $\begin{array}{l}\text { Estado de } \\
\text { México }\end{array}$ & Tlalnepantla de Baz & $114,175.2$ & 51.42 & $\begin{array}{c}\text { PRI-PVM- } \\
\text { N.Alianza }\end{array}$ \\
\hline 5 & $\begin{array}{l}\text { Estado de } \\
\text { México }\end{array}$ & Tianguistenco & $108,885.04$ & 49.04 & PAN-PT \\
\hline 6 & Nayarit & Bahía de Banderas & $103,193.97$ & 46.47 & PRI \\
\hline 7 & Coahuila & Sacramento & $101,200.00$ & 45.58 & PRI \\
\hline 8 & $\begin{array}{l}\text { Estado de } \\
\text { México }\end{array}$ & Villa Guerrero & $99,569.8$ & 44.84 & PRD \\
\hline 9 & $\begin{array}{l}\text { Estado de } \\
\text { México }\end{array}$ & Naucalpan de Juárez & $89,000.01$ & 40.08 & PAN \\
\hline 10 & Nayarit & Tuxpan & $88,444.98$ & 39.83 & PRI \\
\hline
\end{tabular}

Fuente: elaboración propia con base en las páginas de Internet de los municipios en sus secciones de transparencia, según la disponibilidad de información señalada en el cuadro 6.

Destaca el caso de Nayarit, donde los presidentes municipales reciben en promedio más de 56 mil pesos mensuales, y que concentra a 4 de los 10 presidentes con mayores salarios, que ascienden entre los 88 mil y los 121 mil pesos mensuales (cuadro 8). Cabe señalar que no hay una coincidencia entre los partidos políticos que encabezan los gobiernos municipales y las asignaciones salariales; es decir, la discrecionalidad en las asignaciones salariales no tiene sello partidista, sino que atraviesa a todas las fuerzas políticas.

Respecto al caso de Veracruz, es difícil obtener conclusiones contundentes por sólo haber sido posible acceder a poco más del 20\% de los datos sobre remuneraciones.

Ambas dimensiones en juego: cumplimiento de la norma en la integración de los ayuntamientos y remuneraciones

Si se cruza la dimensión del cumplimiento de la norma sobre integración de los ayuntamientos y la dimensión de remuneraciones, se pueden 
Esta revista forma parte del acervo de la Biblioteca Jurídica Virtual del Instituto de Investigaciones Jurídicas de la UNAM

observar coincidencias interesantes, lo que arroja luz sobre las razones detrás de la discrecionalidad y las mecánicas que la mueven.

En primer lugar, respecto de la discrecionalidad en la integración de los ayuntamientos y su relación con la transparencia de las remuneraciones, cabe señalar que los municipios de Veracruz destacan tanto por rebasar el número de integrantes como por ser opacos en lo que se refiere a los salarios de los ediles.

Respecto a los municipios que sí permiten el acceso, se encontró que los regidores que pertenecen a los municipios que se apegan a la norma, reciben una remuneración menor a la de aquellos que laboran en donde - ésta se transgrede. Así, la arbitrariedad en la integración y las remuneracio- nes abultadas parecen ir de la mano. En los casos de los municipios que se apegan a la norma para su integración, los regidores reciben un promedio de 26,713 pesos brutos mensuales, y en los casos de los que no lo hacen, los regidores reciben un promedio de 30,429 pesos brutos mensuales.

Además, en promedio, en aquellos municipios donde se rebasa el número de regidores previstos en la norma, el presidente municipal percibe un sueldo mayor al de los presidentes de los municipios donde ésta se cumple. En los municipios veracruzanos donde se cumple la norma, el presidente municipal gana aproximadamente 15.6 salarios mínimos, mientras que donde se transgrede percibe más de 19 (gráfica 2a).

\section{Gráfica 2. Distribución de las remuneraciones}

DEL PRESIDENTE MUNICIPAL Y SÍNDICOS SEGÚN EL CUMPLIMIENTO DE LA NORMA EN EL NOMBRAMIENTO DE REGIDORES
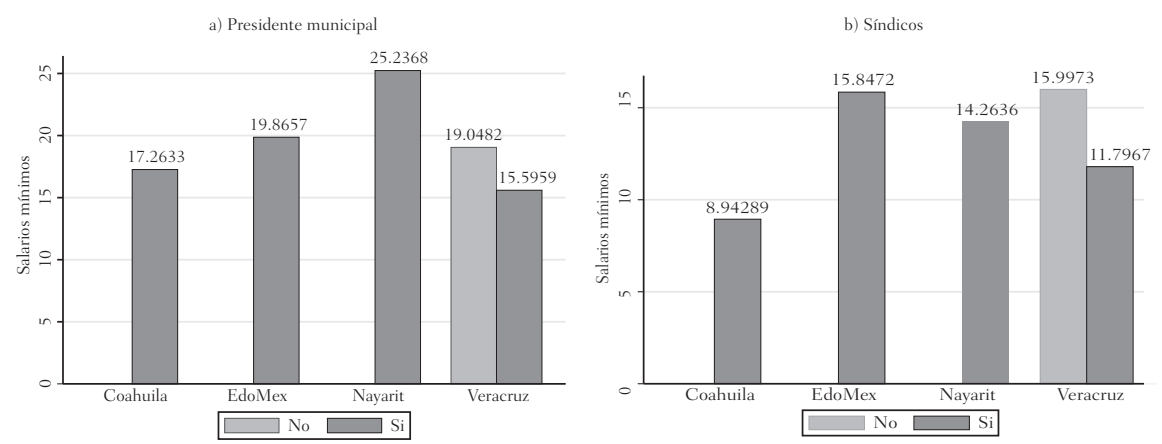

FuENTE: elaboración propia con base en las páginas de Internet de los municipios en sus secciones de transparencia, según la disponibilidad de información señalada en el cuadro 6. 
Esta revista forma parte del acervo de la Biblioteca Jurídica Virtual del Instituto de Investigaciones Jurídicas de la UNAM

Algo similar sucede si analizamos las percepciones que reciben los síndicos: en aquellos municipios donde se excede el número de regidores que establece la norma, los síndicos ganan casi 16 salarios mínimos, mientras que en aquellos donde ésta se observa, su salario equivale a 11.7 salarios mínimos (gráfica 2b).

Un elemento final a destacar, resultado del cruce de las dos dimensiones que interesan a este estudio, es que en los municipios donde se rebasa la proporción de regidores establecida por los códigos electorales estatales respectivos, los regidores reciben un salario promedio mayor $(22,390$ pesos) a los regidores de los municipios en donde se respeta la proporción de MR y RP para la elección de estos funcionarios (18,836 pesos). Así, puede afirmarse que ambas dimensiones avanzan en paralelo (gráfica 3).

\section{Gráfica 3. Salario promedio neto de regidores} POR CUMPLIMIENTO DE NORMA ELECTORAL RESPECTO

A LA DistribuCión DE MR y RP

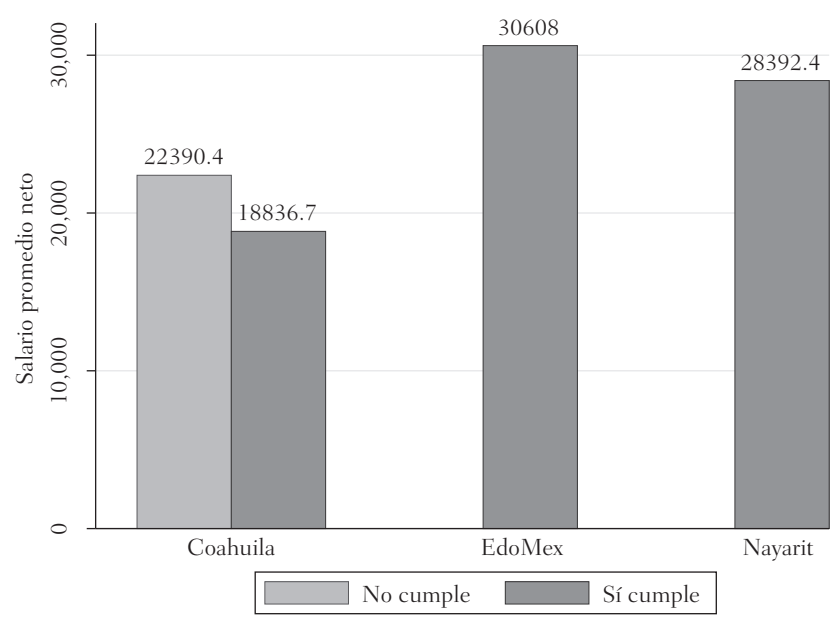

FuEnTE: elaboración propia con base en las páginas de Internet de los municipios en sus secciones de transparencia, según la disponibilidad de información señalada en el cuadro 6.

\section{Consideraciones finales}

A quince años de que las leyes de transparencia y acceso a la información entraran en vigor en todo el país, aparece un buen número de asuntos 
Esta revista forma parte del acervo de la Biblioteca Jurídica Virtual del Instituto de Investigaciones Jurídicas de la UNAM

pendientes que resolver, especialmente en el nivel municipal, donde los obstáculos inician desde la inexistencia de portales electrónicos para consultar información sobre el gobierno hasta el propio procesamiento de las solicitudes de información.

En este trabajo se encontró que 77 municipios (19.4\%) de los 395 contemplados en este estudio no cumplen con la obligación de poseer una página de Internet. De los que sí cuentan con ella, el 69.8\% muestra información básica sobre las remuneraciones de los integrantes de los ayuntamientos. La exigencia de transparentar el ejercicio de todos los recursos públicos para obligar a los servidores públicos a cumplir con lo establecido en - la Constitución y en las leyes, encuentra mayores obstáculos en los munici- pios pequeños (con población menor a los 70 mil habitantes). Los municipios pequeños son los más opacos y, por tanto, es donde tienen lugar los mayores márgenes de discrecionalidad en la asignación de salarios.

Los principios de la transparencia siguen sin arraigar en los municipios. Por ejemplo, en Tultitlán (Estado de México), la información de las remuneraciones de los integrantes del ayuntamiento está clasificada como reservada, y en Tequixquiac (Estado de México), dicha información es inexistente porque los servidores públicos no tienen sueldos, sino dietas. ${ }^{7}$ Además, uno de los argumentos más esgrimidos por 85 de 136 municipios veracruzanos con página de Internet, pero sin información de remuneraciones, es el proceso de migración de la información a la plataforma nacional de transparencia. La transparencia no termina de institucionalizarse en los municipios, y éstos tampoco tienen mayores incentivos para combatir la opacidad.

Más aún, el análisis mostró que los municipios que transgreden la norma en cuanto a integración de los municipios también coinciden en ser los más opacos en remuneraciones. Esta coincidencia, que destaca para los municipios de Veracruz, puede deberse a la laxitud de la norma, pero también a factores institucionales y estructurales más amplios, como las capacidades para atender las obligaciones de transparencia y el nivel extendido de acceso a Internet. Por otro lado, los municipios que permitieron acceder a datos sobre remuneraciones coinciden, en su mayoría, con observar la norma en cuanto a la integración; ser exhibidos en la vitrina pública no parece ser factor suficiente para evitar remuneraciones excesivas e injustificadas. Esto lleva a proponer que las directrices de transparencia deberían de acompañarse de políticas integrales que fortalezcan las capa-

7 Estas respuestas se obtuvieron del procesamiento de las solicitudes 00196/TULTI TLA/IP/2016 y 00024/TEQUIXQU/IP/2016. 
Esta revista forma parte del acervo de la Biblioteca Jurídica Virtual del Instituto de Investigaciones Jurídicas de la UNAM

cidades institucionales para cumplir con los mandatos de transparencia y el acceso de la ciudadanía a información relevante, y de esta manera fortalecer su posibilidad de exigencia y vigilancia social.

Carecer de información accesible sobre la integración y las remuneraciones de los integrantes de los ayuntamientos, datos básicos para cualquier ejercicio de vigilancia ciudadana, impide llevar a cabo ejercicios de monitoreo y seguimiento de la administración municipal, y, por ende, cierra la puerta a exigir cuentas sobre su gestión. Las virtudes del municipio no podrán trascender el plano teórico sin transparencia, uno de los ingredientes fundamentales e imprescindibles para hacer de éste un nivel de gobierno realmente cercano y sensible a las demandas ciudadanas.

\section{Fuentes de INFORMACIÓN}

Carbonell, José, 2002, "Transición a la democracia, gobernabilidad y federalismo en México. Cerrando el círculo”, en Serna, José María (ed.), Federalismo y regionalismo, México, UNAM, Instituto de Investigaciones Jurídicas.

Carbonell, Miguel, 2003, "El federalismo en México: principios generales y distribución de competencias", Anuario de Derecho Constitucional Latinoamericano, UNAM, Instituto de Investigaciones Jurídicas.

Casar, Ma. Amparo, 2010, Sistema político mexicano, México, Oxford University Press.

Cejudo, Guillermo y Ríos, Alejandra, 2010, "La rendición de cuentas del gobierno municipal”, en Merino, Mauricio et al., La estructura de la rendición de cuentas en México, México, UNAM-Centro de Investigación y Docencia Económicas.

Consejo Nacional de Evaluación de la Política Pública de Desarrollo Social (Coneval), 2016, Información de pobreza y evaluación en las entidades federativas, México, disponible en: www.coneval.org.mx/coordi nacion/entidades/Paginas/inicioent.aspx.

DíAz, Alberto, 2002, "Federalismo", Nexos, enero, disponible en: www.nexos. com.mx/?p=10281.

ElazAr, Daniel J., 1997, "Contrasting Unitary and Federal Systems", International Political Science Review, vol. 18, núm. 3.

Fraga, Gabino, 1987, Derecho administrativo, México, Porrúa.

Guillén, Tonatiuh, 2016, Federalismo, gobiernos locales y democracia, México, Instituto Nacional Electoral. 
Esta revista forma parte del acervo de la Biblioteca Jurídica Virtual del Instituto de Investigaciones Jurídicas de la UNAM

Hurtado, Javier, 2012, Representación política y municipio en México. El caso de la capital de Durango, México, Tribunal Electoral del Poder Judicial de la Federación.

Instituto Mexicano para la Competitividad Económica (IMCO), 2016, Índice de información presupuestal municipal, México, disponible en: http://imco.org.mx/wp-content/uploads/2016/12/2016-IIPM-Presentacion. $p d f$.

Instituto Nacional de Estadística y Geografía (INEGI), 2016, Encuesta nacional sobre disponibilidad y uso de TIC en hogares, disponible en: http://www.beta.inegi.org.mx/proyectos/enchogares/regulares/dutih/2016/.

Instituto Nacional de Estadística y Geografía (INEGI), 2015a, Encuesta intercensal 2015, México, disponible en: www.beta.inegi.org.mx/ proyectos/enchogares/especiales/intercensal/.

Instituto Nacional de Estadística y Geografía (INEGI), 2015b, Cuéntame, disponible en: http://cuentame.inegi.org.mx/monografias/default. aspx?tema $=$ me.

Instituto Nacional de Estadística y Geografía (INEGI), 2015c, Resultados del censo nacional de gobiernos municipales y delegacionales, México, disponible en: www.beta.inegi.org.mx/proyectos/censosgobierno/municipal/ cngmd/2015/.

Instituto Nacional para el Federalismo y el Desarrollo Municipal (Inafed), 2012, Los municipios en México: facultad reglamentaria, México, Secretaría de Gobernación.

Instituto Nacional para el Federalismo y el Desarrollo Municipal (Inafed), 2016, Directorios municipales, México, disponible en: www. snim.rami.gob.mx.

Lujambio, Alonso, 1995, Federalismo y congreso en el cambio político de México, México, UNAM.

Lujambio, Alonso, 2000, El poder compartido, un ensayo sobre la democratización mexicana, México, Océano.

Merino, Mauricio, 1992, Fuera del centro, México, Universidad Veracruzana.

Merino, Mauricio, 1996, "Hacia la (re)construcción del federalismo", Nexos, enero, disponible en: www.nexos.com.mx/?p=7692.

Merino, Mauricio, 2017, "Transparencia, rendición de cuentas y combate a la corrupción en los municipios mexicanos: hacia un modelo homogéneo", en Merino, Mauricio et al., Transparencia, rendición de cuentas y combate a la corrupción. El municipio en la encrucijada, México, CIDE. 
Esta revista forma parte del acervo de la Biblioteca Jurídica Virtual del Instituto de Investigaciones Jurídicas de la UNAM

Meyer, Lorenzo, 1994, "El municipio mexicano al final del siglo XX. Historia, obstáculos y posibilidades", en Merino, Mauricio (coord.), En busca de la democracia municipal, México, El Colegio de México.

PARDo, María del Carmen, 1994, "Gestión municipal ¿motor o freno para el ejercicio democrático?”, en Merino, Mauricio (coord.), En busca de la democracia municipal, México, El Colegio de México.

Pérez, Jorge, 2011 , "Alcalde de Tlalnepantla es el que mejor gana del país", Proceso, México, disponible en: www.proceso.com.mx/287582/alcalde-detlalnepantla-es-el-que-mejor-gana-del-pais-749-mil-al-mes.

Riker, William, 1975, "Federalism”, en Greenstein, Fred y Polsby, Nelson (eds.), Handbook of Political Science, Reading, Massachussets, AddisonWesley.

RolDÁn, José, 2013, La rendición de cuentas y responsabilidad por actividad discrecional y por omisión, México, Red por la Rendición de CuentasCIDE.

Secretaría de Desarrollo Social (Sedesol), 2010, Organización del cabildo, México, Banco Mundial-Sedesol.

UGALDE, Luis Carlos, 2002, La rendición de cuentas en los gobiernos estatales y municipales, México, Auditoría Superior de la Federación.

\section{Marco jurídico}

Código Electoral para el Estado de Coahuila de Zaragoza (CEEC), disponible en: http://congresocoahuila.gob.mx/portal/?page_id=538.

Código Electoral del Estado de México (CEEM), disponible en: http://legis lacion.edomex.gob.mx/node/2147.

Código Electoral para el Estado de Veracruz de Ignacio de la Llave (CEEV), disponible en: www.legisver.gob.mx/Inicio.php? $p=c o$.

Constitución Política del Estado de Coahuila de Zaragoza (CPCZ), disponible en: http://congresocoahuila.gob.mx/portal/wp-content/uploads/2014/11/ coa01.pdf.

Constitución Política del Estado de Veracruz de Ignacio de la Llave (CPEV), disponible en: www.legisver.gob.mx/leyes/ConstitucionPDF/CONSTITU CION0407172.pdf.

Constitución Política del Estado Libre y Soberano de México (CPELSM), disponible en: http://legislacion.edomex.gob.mx/constitucion_local.

Constitución Política del Estado Libre y Soberano de Nayarit (CPELSN), 
Esta revista forma parte del acervo de la Biblioteca Jurídica Virtual del Instituto de Investigaciones Jurídicas de la UNAM

disponible en: www.congresonayarit.mx/qué-hacemos/compilación-legisla tivalconstitución-pol\%C3\%ADtica-del-estado-libre-y-soberano-de-nayarit/.

Constitución Política de los Estados Unidos Mexicanos (CPEUM), disponible en: www.diputados.gob.mx/LeyesBiblio/index.htm.

Ley Electoral del Estado de Nayarit (LEEN), disponible en: http://ieenaya rit.org/html/legislacion.html.

26 Ley General de Transparencia y Acceso a la Información Pública (LGTAIP), disponible en: www.diputados.gob.mx/LeyesBiblio/pdf/LGTAIP.pdf.

Ley Orgánica Municipal del Estado de México (LOMEM), disponible en: www.secretariadeasuntosparlamentarios.gob.mx/leyes_y_codigos.html.

- Ley Orgánica del Municipio Libre del Estado de Veracruz (LOMLEV), disponible en: www.legisver.gob.mx/Inicio.php? $p=l e$.

Tesis P./J. 8/2010, Semanario Judicial de la Federación y su Gaceta, Novena Época, Pleno, t. XXXI, febrero de 2010.

Tesis P./J. 19/2013, Semanario Judicial de la Federación y su Gaceta, Décima Época, Pleno, libro XX, mayo de 2013. 